\title{
Instantaneous phase mapping deflectometry for dynamic deformable mirror characterization
}

Isaac Trumper, Heejoo Choi, Dae Wook Kim

Isaac Trumper, Heejoo Choi, Dae Wook Kim, "Instantaneous phase mapping deflectometry for dynamic deformable mirror characterization," Proc. SPIE 10401, Astronomical Optics: Design, Manufacture, and Test of Space and Ground Systems, 104010S (5 September 2017); doi:

$10.1117 / 12.2272188$

SPIE Event: SPIE Optical Engineering + Applications, 2017, San Diego, California, United States 


\title{
Instantaneous phase mapping deflectometry for dynamic deformable mirror characterization
}

\author{
Isaac Trumper ${ }^{\mathrm{a}}$, Heejoo $\mathrm{Choi}^{\mathrm{a}}$, and Dae Wook Kimª \\ ${ }^{a}$ College of Optical Sciences, University of Arizona, 1630 E. University Blvd., Tucson, AZ \\ 85721, USA

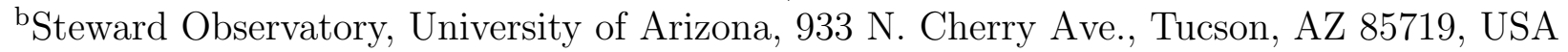

\begin{abstract}
We present an instantaneous phase mapping deflectometry (PMD) system in the context of measuring a continuous surface deformable mirror (DM). Deflectometry has a high dynamic range, enabling the full range of surfaces generated by the DM to be measured. The recent development of an instantaneous PMD system leverages the simple setup of the PMD system to measure dynamic objects with accuracy similar to an interferometer. To demonstrate the capabilities of this technology, we perform a linearity measurement of the actuator motion in a continuous surface DM, which is critical for closed loop control in adaptive optics applications. We measure the entire set of actuators across the DM as they traverse their full range of motion with a Shack-Hartman wavefront sensor, thereby obtaining the influence function. Given the influence function of each actuator, the DM can produce specific Zernike terms on its surface. We then measure the linearity of the Zernike modes available in the DM software using the instantaneous PMD system. By obtaining the relationship between modes, we can more accurately generate surface profiles composed of Zernike terms. This ability is useful for other dynamic freeform metrology applications that utilize the DM as a null component.
\end{abstract}

Keywords: Deflectometry, Deformable Mirror, Dynamic Metrology

\section{INTRODUCTION}

We present results of a linearity measurement of a continuous surface deformable mirror (DM) using an instantaneous phase shifting deflectometry system implemented on an iPhone ${ }^{\circledR}$. This technology enables snapshot phase shifting deflectometry data acquisition, measurements in high vibration environments, and a host of other scenarios inaccessible to conventional phase shifting deflectometry techniques. We accomplish the instantaneous measurement with a multiplexed display pattern and novel data processing*, such that we can play back a video of time varying events in the measurement path. ${ }^{1}$

\subsection{Deflectometry Background}

Deflectometry is a surface slope measuring tool that requires minimal hardware and acquires surface height data with nanometer-level precision. ${ }^{2}$ It directly measures slope data, and has a very large dynamic range. ${ }^{3}$ At the most basic level, a deflectometry system must have a screen to display a pattern, and a camera to capture images of the mirror under test, which is illuminated by the screen. A schematic of the measurement setup is shown in Fig. 1. The camera hardware can be chosen from off-the-shelf components where low signal-to-noise and fast acquisition times are desirable. The screens can also be off-the-shelf, but the patterns displayed are areas of active research. The camera is positioned such that it focuses on the mirror's surface while close to the center of curvature of the optic. For a flat mirror, as shown in Fig. 1, the axial location is primarily determined by sampling criteria. In practice, the camera must be shifted off-axis in order to collect light from the screen that is reflected off of the test optic. It is therefore helpful to conceptualize the geometry by tracing rays from the

\footnotetext{
Further author information: (Send correspondence to D.W.K.)

D.W.K.: E-mail: letter2dwk@hotmail.com

I.T.: E-mail: itrumper@optics.arizona.edu

H.C.: E-mail: hchoi@optics.arizona.edu

${ }^{*}$ Most of section 1 is adapted from a previous publication by Trumper ${ }^{1}$
} 


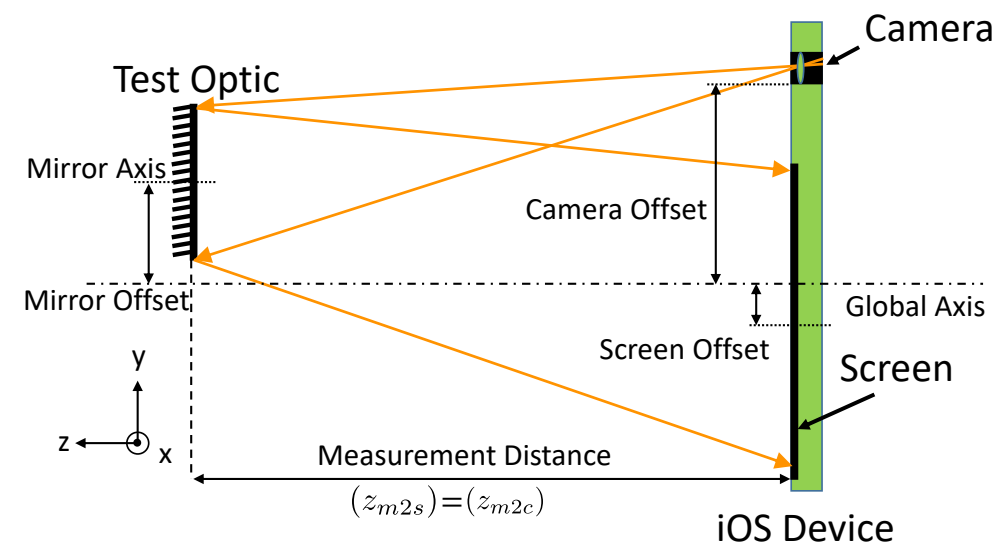

Figure 1. Schematic of a typical deflectometry measurement with all relevant distances indicated for use with a mobile device.

camera to the screen to determine where they intersect the mirror and screen. The camera acts as our eye, and each pixel on its detector will correspond to a point on the mirror and screen. These three points in 3D space, across the entire optic's surface, define the knowledge required to perform a measurement. One should think of this as a mapping between the camera and screen caused by the optic.

To create a mapping, current deflectometry systems use display patterns such as line-scanning, binary patterns, and phase shifting. ${ }^{4}$ The general process of creating a mapping using the phase of a pattern is defined by the authors as Phase Mapping Deflectometry (PMD). This encompasses and generalizes the well-established Phase Shifting Deflectometry (PSD) systems while providing a more accurate naming convention. A phase shifting approach is utilized in the following work. To acquire the slope data, we first display a sinusoidal pattern across the screen of a fixed frequency by modulating the output brightness of individual pixels. We then shift the pattern by a fixed phase shift until we have completed a full $2 \pi$ phase shift. This pattern is displayed in both $x$ and $y$ directions separately from one another. After collecting the images corresponding to the phase shifted display pattern, we apply a phase shifting algorithm ${ }^{5}$ to obtain wrapped mapping data. The wrapped mapping is unwrapped using typical methods found in interferometry, ${ }^{6}$ which results in the final mapping between the camera and screen through the surface under test. We then perform a slope calculation, given by Equation 1, to obtain the local slope at every sample point across the surface. Note that Equation 1 is for the $x$-direction slopes, while the $y$-slopes are computed using the same form of equation, but with the corresponding $y$-direction variables.

$$
s_{x}=\frac{1}{2}\left(\frac{x_{m}-x_{s}}{z_{m 2 s}}+\frac{x_{m}-x_{c}}{z_{m 2 c}}\right),
$$

where $s_{x}$ is the local slope, $x_{m}$ is the local mirror surface coordinate, $x_{s}$ is the screen pixel coordinate, $x_{c}$ is the camera pixel coordinate, $z_{m 2 s}$ is the distance from the mirror to the screen, and $z_{m 2 c}$ is the distance from the mirror to the camera. We must then integrate the slope data to obtain surface height information, which we accomplish with either a pixel-by-pixel (zonal) method or by fitting with analytic functions (modal).

\subsection{Instantaneous Phase Mapping Deflectometry}

All previously investigated phase shifting methods rely on changing the pattern with time and recording multiple images with the camera to reconstruct the optical surface under test. These methodologies cannot cope with time varying measurements because they multiplex information in the time domain. In doing this, they are limited to measurements in which the environment, or features on the surface, do not change in time. In our instantaneous PMD, we do not encounter such limitations because we multiplex all the necessary information into a single screen and capture it with a single snapshot.

The phase shifting method of multiplexing incorporates two main ideas, each with an analogous concept in the realm of instantaneous interferometry. First, we encode the phase information using color, which requires a color display and camera. This style of registration is similar to that employed by the polarization multiplexed 
interferometer, where each phase shift is detected independently from the others. The number of color channels is fixed by the hardware, so we use a three-step phase shifting algorithm, the minimum required. Second, we display a large number of fringes on the screen, which acts as a carrier frequency in the image, similar to the spatial frequency carrier interferometer. When we combine both of these tools, we are able to distinctly multiplex six pieces of information corresponding to the three phase shifts in the two orthogonal directions necessary for a phase shifted deflectometry measurement. It is important to note that the analogies given here are only meant to provide an intuition into the multiplexing methods for those more familiar with interferometry. The comparison should not be understood as implying that the interferometry methods are the same as the deflectometry methods because the two metrology systems operate on fundamentally different principles.

For convenience, we define the two orthogonal directions of the fringes to be in the $x$ and $y$ directions, which lie in the plane of the screen as shown in Fig. 1. They are able to be in any orientation, but this coordinate system is best for displaying fringes accurately. The $x$ and $y$ fringe data must be displayed simultaneously, resulting in a pattern that looks more like an oscillating membrane than fringes. Furthermore, each phase shift is superimposed, so the resulting display is a multicolored membrane that does not resemble traditional fringes. This pattern is shown at the start of the data flow chart, given in Fig. 2, labeled as 'Display Image'.

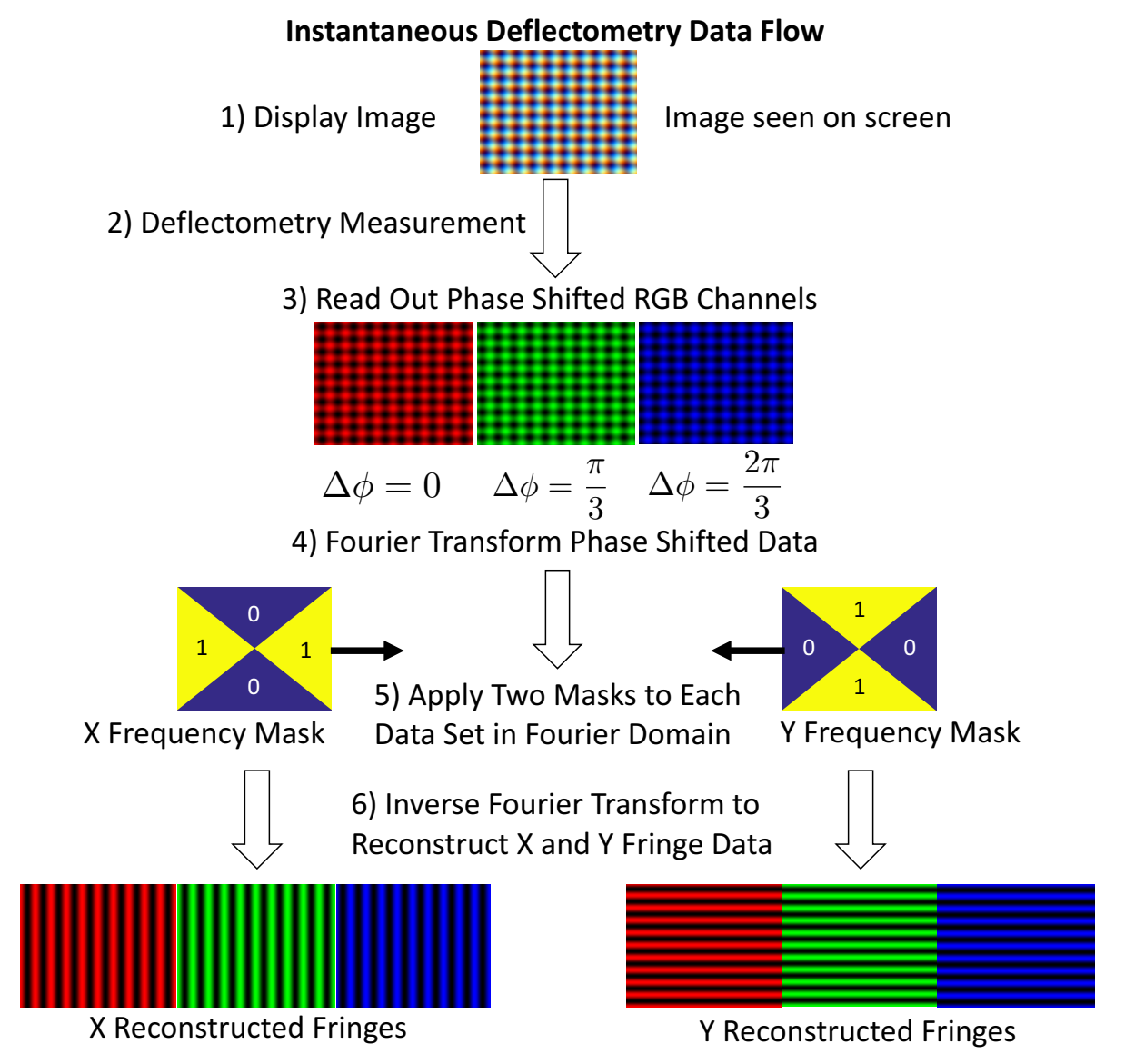

Figure 2. Overview of the instantaneous phase mapping multiplexing method and their incorporation a deflectometry measurement. ${ }^{1}$ The images shown are all synthetically generated with MATLAB ${ }^{\circledR}$. Note that the data processing does not end at the last step shown, further phase unwrapping and integration steps are required.

The camera captures an image of the surface under test, which is illuminated by the screen, and the resulting image is a distorted version of the display image. The camera has three color channels which are read out separately to obtain three sets of data, corresponding to the three phase shifts: red $(\Delta \phi=0)$, green $\left(\Delta \phi=\frac{\pi}{3}\right)$, and blue $\left(\Delta \phi=\frac{2 \pi}{3}\right)$. We then Fourier transform each phase shifted image. In the Fourier domain, we observe 
distinct peaks at the locations corresponding to the carrier frequencies of the display fringes in the $x$ and $y$ directions. By dividing the Fourier domain into $x$ and $y$ frequencies without rejecting any information, we are able to decompose the input image into two separate images that would have been observed had we displayed one directional fringes. The separation boundaries applied to the frequency data in instantaneous phase shifting deflectometry are shown in Fig. 2 labeled as 'X Frequency Mask', and 'Y Frequency Mask'. We call them bow tie and hour glass masks, respectively, due to their shape. In practice, due to discrete sampling, the edge boundary is actually jagged, but in an analytic case it would be smooth. Also, the center pixel (zero frequency) is used in both masks to preserve the mean intensity value. The masks separate out a single frequency direction, while preserving the details of the fringe pattern contained in each frequency direction. They work on the principle that with a sufficiently large carrier frequency in the display membrane, or a dense fringe pattern, the component $x$ and $y$ fringes are distinguished with high fidelity in the Fourier domain. We then apply an inverse Fourier transform to the separated data and reconstruct the one directional fringe patterns that made up the input image. From the single input image, we are able to obtain six unique outputs that comprise the three phase shifts in both orthogonal directions required to reconstruct the surface under test.

Using the instantaneous method we are able to measure a dynamically varying surface and reconstruct the surface for every image captured. We employ a relative measurement technique that measures the change in the surface from a nominal state. This enables a high level of systematic error correction to achieve accuracies similar to interferometry. ${ }^{1}$

\section{EXPERIMENTAL SETUP}

\subsection{Deformable Mirror}

A deformable mirror (DM) is a useful component in many systems that require dynamic optical surfaces such as adaptive optics in astronomy ${ }^{7}$ where they are used to correct time varying atmospheric aberrations. Using the instantaneous PMD system developed on the iPhone ${ }^{\circledR}$, we tested a DM produced by ALPAO on its ability to linearly combine Zernike terms when creating a time varying surface. The specifications for the DM that we used in our experiment are listed in Table 1.

Table 1. Relevant specifications of the deformable mirror used to generate the surface measured using instantaneous phase mapping deflectometry.

\begin{tabular}{ccccc} 
Model & Pitch & \# of Actuators & Diameter & Settling Time (at $\pm 5 \%)$ \\
\hline DM52-25 & $2.5 \mathrm{~mm}$ & 52 & $15 \mathrm{~mm}$ & $2.0 \mathrm{~ms}$
\end{tabular}

To test the linearity of the DM, we input a command to produce a specific Zernike surface, measuring the surface as a function of time as the input coefficient increases and decreases linearly in magnitude. We then make a second measurement of a different Zernike surface and finally a third measurement of the linear combination of the first two surfaces. With these three data sets, we can examine how the DM responds to the linear combination of two Zernike terms. We chose to test Zernike terms corresponding to primary astigmatism (Z5) and secondary astigmatism $(\mathrm{Z} 12) .^{8}$

The DM was calibrated prior to the experiment using a Shack-Hartmann wavefront sensor (SHWS) in order to provide the software with a look-up table to produce the required Zernike terms. The SHWS was used because it provided a direct method of inputing the calibration data into the DM's software. Note that because this calibration process has its own sources of error, we are not testing the DM's ability to produce pure Zernike surfaces, but rather the linear combination of the surfaces created.

\subsection{Test Geometry}

The measurement geometry is shown in Fig. 3, where the iPhone ${ }^{\circledR}$ is held in 3D printed mount and placed approximately $10 \mathrm{~cm}$ away from the DM's surface. We measure the DM surface at a rate of $10 \mathrm{~Hz}$, limited by how quickly the iPhone can capture image data and save to a file at its highest resolution settings. A total of 75 sequential images are collected for each experiment, yielding a measurement interval of 7.5 seconds over which we actuate the DM. 


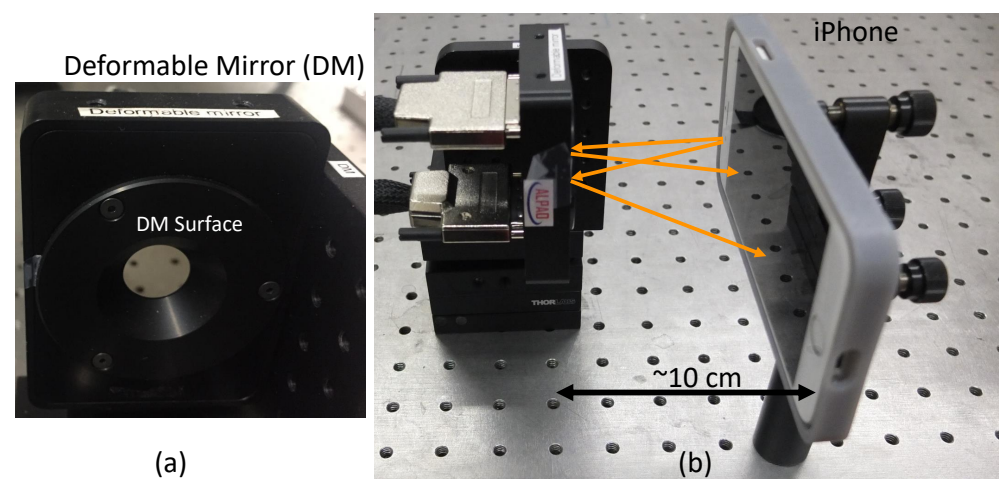

Figure 3. Experimental setup of the measurement featuring the iPhone and deformable mirror. (a) The DM's 15 mm circular surface, which is obscured from view in (b). Shown in (b) are orange arrows that approximate the time-reversed paths of the rays from the front-facing camera of the iPhone reflected off the mirror's surface and striking the iPhone's screen, similar to those shown in Fig. 1.

\section{LINEARITY MEASUREMENT RESULTS}

We process the recorded images using the instantaneous scheme depicted in Fig. 2, producing surface height data across the DM's $15 \mathrm{~mm}$ circular aperture. The three distinct Zernike surfaces we measured are shown in Fig. 4a-c next to the surface (Fig. 4d) showing the synthetic combination of the two individual Zernike surfaces (Fig. 4ab) to compare against the generated (and measured) combination of the two terms (Fig. 4c). As described previously, we are not testing the DM's ability to generate the specified Zernike term because the calibration procedure using the SHWS is not perfect. Therefore, the non-pure Zernike surfaces shown in Fig. 4a-b are tolerated. We see a discrepenacy between the synthetic linear combination and the measured linear combination due to errors in generating the combination of the Zernike surfaces. However, due to the implementation on the iPhone and controlling the DM via a separate computer, we are not able to precisely time each measurement, leading to small temporal offsets between the measurements that could also cause differences seen between Fig. 4c and d. To compensate as much as possible for this effect, we examined each data from the three experiments and aligned the start of the DM motion to take place at the same frame in each data.

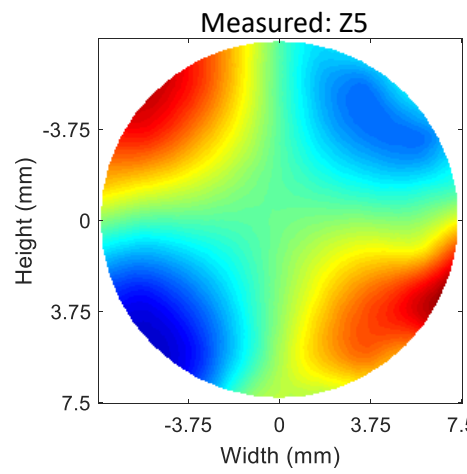

(a)

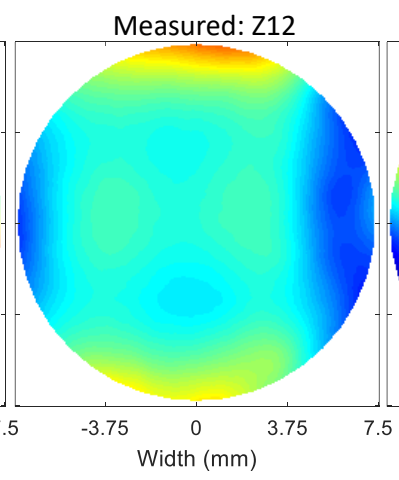

(b)

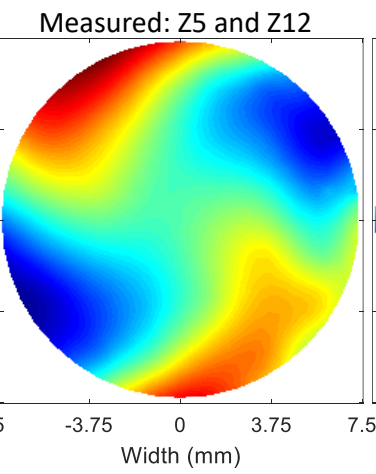

(c)

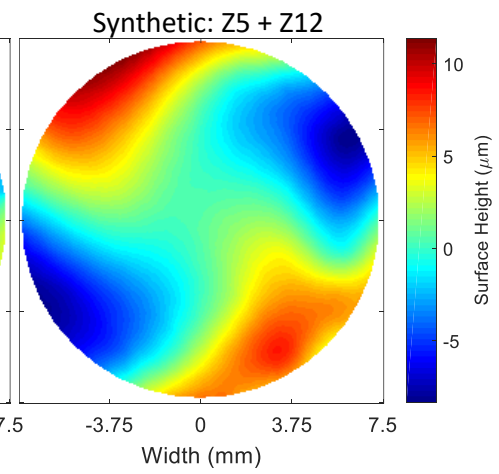

(d)

Figure 4. Measured surfaces of the deformable mirror used to investigate the DM's linearity along with a link to the full video file from which the surface data was taken. (a) Zernike coefficient Z5: http://dx.doi.org/doi.number. goes.here (b) Zernike coefficient Z12: http://dx.doi.org/doi.number.goes.here (c) Zernike coefficients Z5 and Z12 simultaneously: http://dx.doi.org/doi.number.goes.here and (d) Sum of the results in (a) and (b): http://dx.doi. org/doi.number.goes.here. Note the discrepancy between (c) and (d), either due to the deformable mirror's inability to linearly combine the Zernike surfaces or mistiming between the measurements. Also note that all figures are plotted on the same scale.

By fitting the measured surfaces with Zernike coefficients (standard ordering), we are able to see how the 
value of the coefficient (RMS surface contribution) varies as a function of time between the synthetic linear combination and the measured linear combination. We expect a linear change in the coefficient up to a peak or valley (depending on the sign), and then back to its starting value of zero. Shown in Fig. 5 are the plots of this behavior corresponding to the two Zernike coefficients that we selected along with the RMS difference between the measured surface and the synthetically summed surface. The surfaces have other Zernike terms present, but they are not our concern because of the calibration of the DM. For each Zernike coefficient, we plot the measured linear combination (blue line) and the synthetic linear combination (red line) on the same axis for a direct comparison. We see that there is good agreement for each coefficient throughout the full range of coefficient values resulting in RMS differences of $0.25 \mu \mathrm{m}$, and $0.08 \mu \mathrm{m}$, for Z5, and Z12, respectively. The large deviation in the Z12 coefficient (Fig. 5b) is most likely the same difference that we see betwee Fig. 4c-d, where the DM encounters errors generating the linear combination. The change in the coefficient value is also nonlinear for the measured combination while more linear for the synthetic combination, indicating that the DM may generate errors when combining Zernike coefficients. The largest discrepancy between the two data is found at the maximum value of the coefficient (peak or valley), most likely due to the DM reaching its maximum stroke at these locations causing a nonlinear effect. The slope of the change in coefficient is very similar between the two cases, but it is not perfectly linear. This behavior is most likely caused by the errors during calibration with the Shack-Hartman wavefront sensor, but it could also be an issue with the stroke of the DM's actuators.

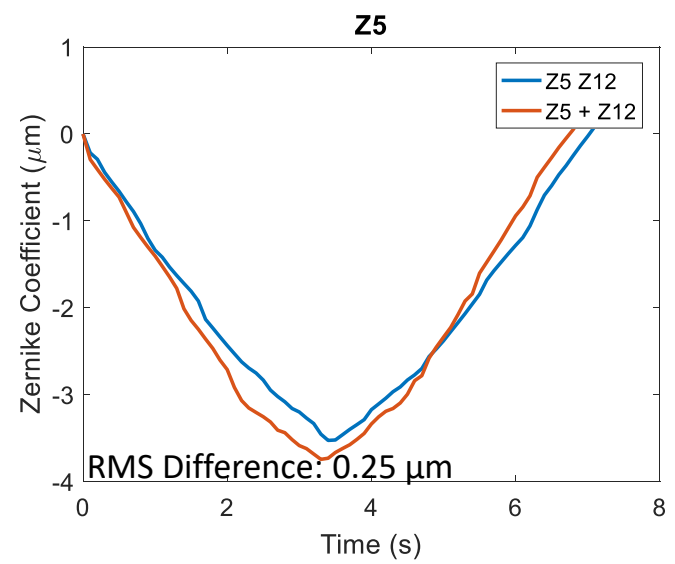

(a)

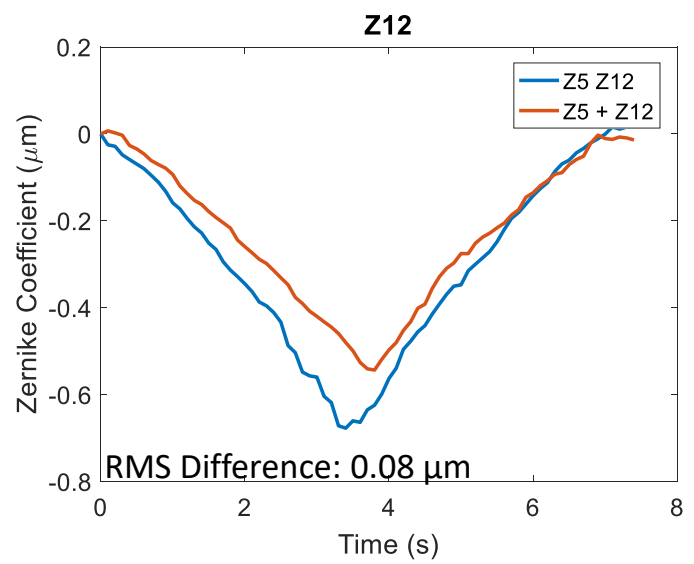

(b)

Figure 5. Value of the specfied Zernike coefficent (RMS contribution) as a function of time showing the linearity of the deformable mirror. The Zernike terms were fit to the measured surface data in two cases. First, when the deformable mirror's surface was a combination of Z5 and Z12 (blue) and second, the sum of the individual surface data from the Z5 and Z12 cases (red). We see a good agreement between the sum and simultaneously generated surfaces, showing that the deformable mirror is a linear system for this combination of Zernike terms.

We further examined the capabilites of the deformable mirror by measuring a surface with eight Zernike modes (Z5, Z6, Z8, Z9, Z11, Z12, Z13, and Z15) multiplexed. This experiment also tests the capabilites of the hardware and software in reconstructing the surface. A typical application of a deformable mirror will utilize a large number of Zernike terms, and therefore it is important that the instantaneous PMD system is sensitive to each term. Shown in Fig. 6(a) is a sample of this measurement, where the surface height change is at its maximum and in (b) is a bar plot of the Zernike term contribution to the surface. We see a large superposition of Zernike terms, even getting nonzero values in modes that were not set by the deformable mirror's software. This is most likely due to a combination of the imperfect DM calibration with the SHWS and the mode cross-talk by the DM.

\section{CONCLUSION}

We demonstrated an application for the instananeous phase mapping deflectometry system implemented on an iPhone ${ }^{\circledR}$, where a deformable mirror was tested for its linearity when generating Zernike surfaces. In this case 


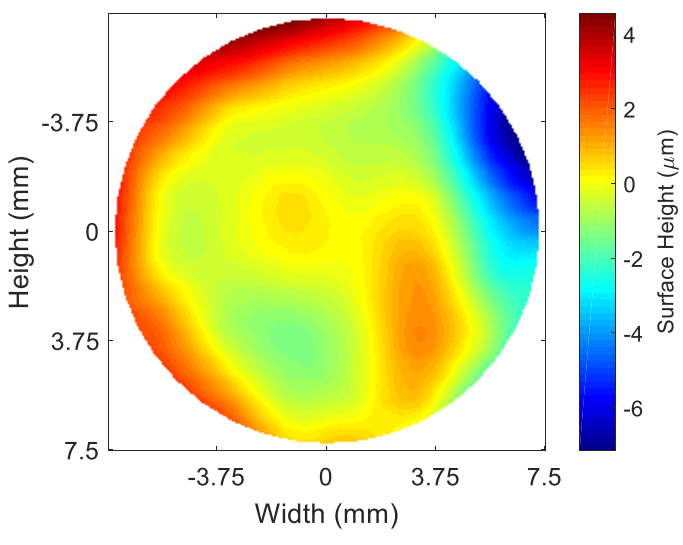

(a)

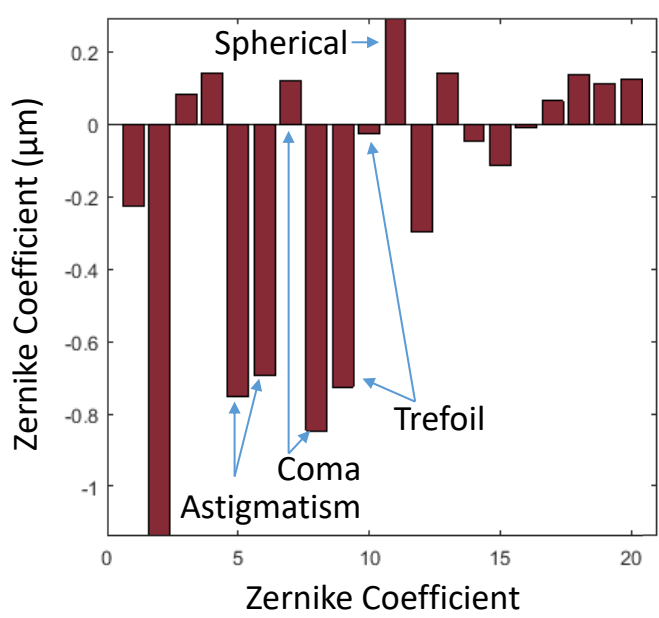

(b)

Figure 6. Measurement of a many-termed Zernike surface generated by the DM, where (a) is the surface reconstruction at the point of maximum surface deviation from nominal, and (b) shows a Zernike mode histogram contributing to the measured surface. Up to Z20 was fit to analyze the surface.

we have demonstrated the capabilites of measuring low-order Zernike surface terms as well as the potential to use as a dynamic calibration tool for the deformable mirror. This characterization and potential calibration of the deformable mirror's surface has applications in adaptive optics where deformable mirrors are used to correct time varying wavefronts. The instantaneous PMD system is well suited for relative dynamic metrology such as tracking the change due to environmental conditions or an actively controlled surface. We hope that this work inspires other uses for this technology and enables further dyanmic metrology of specular surfaces that was not previously possible.

\section{ACKNOWLEDGMENTS}

The authors would like to thank the members of the LOFT group for their support during the development of this technology. This research was made possible in part by the Technology Research Initiative Fund (TRIF) Optics/Imaging Program, through the College of Optical Sciences at the University of Arizona, the Post-processing of Freeform Optics project supported by the Korea Basic Science Institute, and the II-VI Foundation Block-Gift Program for helping support general deflectometry research in the LOFT group.

\section{REFERENCES}

[1] Trumper, I., Choi, H., and Kim, D., "Instantaneous phase shifting deflectometry," Optics Express 24, 2799328007 (2016).

[2] Su, P., Parks, R., Wang, L., Angel, R., and Burge, J., "Software configurable optical test system: a computerized reverse hartmann test," Applied Optics 49, 4404-4412 (2010).

[3] Su, P., Wang, S., Khreishi, M., Wang, Y., Su, T., Zhou, P., Parks, R., Law, K., Rascon, M., Zobrist, T., Martin, H., and Burge, J., "Scots: A reverse hartmann test with high dynamic range for giant magellan telescope primary mirror segments," Proc. SPIE 8450, 1-9 (2012).

[4] Butel, G., Smith, G., and Burge, J., "Optimization of dynamic structured illumination for surface slope measurements," Proc. SPIE 8493, 1-12 (2012).

[5] Malacara, Z. and Servin, M., [Interferogram analysis for optical testing], vol. 84, CRC press (2016).

[6] Asundi, A. and Wensen, Z., "Fast phase-unwrapping algorithm based on a gray-scale mask and flood fill," Applied optics 37(23), 5416-5420 (1998).

[7] Madec, P.-Y., "Overview of deformable mirror technologies for adaptive optics and astronomy," (2012).

[8] Noll, R. J., "Zernike polynomials and atmospheric turbulence*," J. Opt. Soc. Am. 66, 207-211 (Mar 1976). 